\title{
Stochastic Effects on the Dynamics of a Resonant MEMS Magnetometer: a Monte Carlo Investigation
}

\author{
Mehrdad Bagherinia ${ }^{1, *}$, Stefano Mariani ${ }^{1}$ and Alberto Corigliano ${ }^{1}$ \\ 1 Politecnico di Milano, Dipartimento di Ingegneria Civile e Ambientale, Piazza L. da Vinci 32, \\ 20133 Milano, Italy; E-Mails: mehrdad.bagherinia@polimi.it (M.B.); stefano.mariani@polimi.it \\ (S.M.); alberto.corigliano@polimi.it (A.C.) \\ * Author to whom correspondence should be addressed; E-Mail: mehrdad.bagherinia@polimi.it; \\ Tel.:+39-02-23994274; Fax: +39-02-23994300.
}

Published: 1 June 2014

\begin{abstract}
In the design of Lorentz force MEMS magnetometers, the coupled thermoelectro-magneto-mechanical fields governing the dynamics of the relevant compliant structures can be appropriately exploited to enhance their performances. In recent works, we showed that reduced-order models for the dynamics of the said movable structures can be recast in the form of the Duffing equation, where nonlinear terms arise from the multiphysics governing the problem. As stochastic effects may play a role due to the micrometric dimensions of the device, an investigation of the link between the statistics of sensor imperfections and output is here carried out. The said imperfections at the microscopic length-scale are modeled in terms of: overetch thickness, assumed to feature a uniform distribution in a proper interval matching available experimental data; and elastic properties of the vibrating polycrystalline silicon film, as obtained through a numerical homogenization procedure over a representative film volume. To get insights into the effects of the parameters governing the nonlinear dynamics of the resonant structure, a Monte Carlo analysis is adopted.
\end{abstract}

Keywords: MEMS; Lorentz force magnetometer; coupled thermo-electro-magnetomechanical formulation; sensitivity to imperfections. 


\section{Introduction}

Rapid development of semiconductor manufacturing technology has enabled the mass production of micro electrical-mechanical system (MEMS) devices, like e.g. magnetometers. To design and predict the performance of this kind of devices, it is required to accurately model the interaction of various physical fields like the mechanical, thermal, electric and magnetic ones. In recent years, different numerical and analytical methods were proposed to better understand the underlying working principles, and increase the ability to capture the experimental evidence [1-3].

These approaches assume that the geometrical and physical properties of the device are known in a deterministic sense. In reality, significant uncertainties in these properties are unavoidable due to a variety of factors linked to the chemical and physical processes adopted for manufacturing. For example, there could always be a so-called overetch associated with the geometrical features of the sensor structure, like e.g. the width of beams and the relevant gap from the substrate. Material properties, such as the Young's modulus of polycrystalline films, can be another source of uncertainties. As a result, it is imperative to assess the effects of these stochastic variations on various design parameters, or performance indexes through theoretical and/or computational approaches.

The MEMS system considered in this work is a uniaxial capacitive Lorentz force magnetometer. To model its dynamics, a coupled thermo-electro-magneto-mechanical formulation was presented in [2] and is here further discussed, along with a rather standard approach to obtain a relevant reduced-order (1 degree-of-freedom, DOF) model. We then exploit a numerical homogenization procedure to obtain the probability density function (pdf) of the Young's modulus for a representative volume of polycrystalline films. As details of the manufacturing process cannot be directly linked to the pdf of the overetch thickness, the said pdf is assumed to be constrained uniform. A Monte Carlo analysis is then exploited to propagate these uncertainties by means of the proposed multi-physics formulation, specifically focusing on the device sensitivity.

\section{Reduced-order model of the vibrating MEMS structure}

Figure 1. SEM image of the moving structure of the resonant Lorentz force MEMS magnetometer, and relevant reference frame.

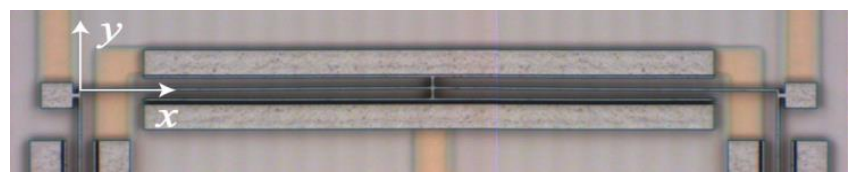

As already reported in [2], part of the resonating structure of the studied Lorentz force MEMS magnetometer is as shown in Figure 1. This structure senses any magnetic field aligned with the outof-plane direction ( $z$, according to the reference frame of Figure 1); sensing is obtained thanks to the two parallel plates attached to the mid-span cross-section of the beam.

The beam, featuring a length $L$ and a cross-section of area $A=b h$, is assumed in a clampedclamped configuration. Its elastic response is modeled according to second-order theory, allowing for lateral deflections small in amplitude but affecting the equilibrium state. Due to the beam slenderness, dynamic equilibrium is enforced in weak form through: 


$$
\int_{0}^{L} \delta v^{\prime \prime} E I v^{\prime \prime} \mathrm{d} x+\int_{0}^{L} \delta v \eta \ddot{v} \mathrm{~d} x-\int_{0}^{L} \delta v^{\prime} P v^{\prime} \mathrm{d} x-\int_{0}^{L} \delta v f \mathrm{~d} x=0
$$

where: $\delta \boldsymbol{\square}$ stands for the variation of field $\boldsymbol{\square} ; v(x, t), t$ being time, is the lateral deflection; $v^{\prime}=$ $\partial v / \partial x$ and $v^{\prime \prime}=\partial^{2} v / \partial x^{2}$ respectively represent the rotation and the curvature of the beam axis; $\ddot{v}=\partial^{2} v / \partial t^{2}$ represents the lateral acceleration field along the beam axis; $E I$ is the flexural rigidity of the beam; $\eta$ is the mass per unit length of the beam; $P$ is the axial compressive load, related to residual stresses; $f$ is the magnitude of the lateral load per unit length, provided by the external actions.

To obtain from Eq. (1) a 1-DOF, reduced-order model of the system, we assume the beam to deform according to its fundamental, linear flexural vibration mode. Due to the clamped-clamped boundary conditions, this mode reads:

$$
v(x, t)=\frac{1}{2}\left(1-\cos \left(\frac{2 \pi x}{L}\right)\right) \mathcal{V}(t)
$$

Here, the variations in space and time are multiplicative decomposed, and $\mathcal{V}$ represents the lateral displacement at the mid-span cross-section. The motion of the beam is then described by:

$$
m \ddot{\mathcal{V}}+d \dot{\mathcal{V}}+K_{1} \mathcal{v}+K_{3} \mathcal{V}^{3}=F
$$

where, see [2]:

$$
\begin{aligned}
m & =\frac{3}{8} \eta L+2 \eta^{*} L \\
d & =\frac{\mu L b^{3}}{8 g^{3}} \\
K_{1} & =\frac{2 \pi^{4}}{L^{3}} E I-\frac{\pi^{2}}{3 L} \alpha E A \Delta T_{m}-2 \frac{\varepsilon_{0} b L}{g^{3}} V_{0}^{2} \\
K_{3} & =\frac{\pi^{4}}{8 L^{3}} E A-4 \frac{\varepsilon_{0} b L}{g^{5}} V_{0}^{2} \\
F & =\frac{L}{2} i B
\end{aligned}
$$

represent the effective mass, damping, linear and cubic stiffness, and external load terms. In Eqs. (4): $\eta^{*}$ is the mass per unit length of the attached sensing plates; $\mu$ is the viscosity coefficient of the air surrounding the resonating structure; $g$ is the gap between the two surfaces of the parallel-plate capacitors; $\alpha$ is the coefficient of longitudinal thermal expansion; $\Delta T_{m}$ is the mid-plan temperature raise due to Joule effect; $V_{0}$ is the driving potential; $\varepsilon_{0}$ is the permittivity of vacuum; $i$ is the density of the current longitudinally flowing in the beam; $B$ is the magnitude of the magnetic field to be sensed.

In the formulation here above, the mass term accounts for the contributions of the beam itself and of the attached plates for sensing. The damping terms is instead coming from the interaction between the plates and the fixed sensing electrodes, leading to a squeeze of the fluid film in between. The three contributions to the term $K_{1}$ are respectively provided by: the bending stiffness of the beam; the softening (hence the minus sign) due to the Joule effect, leading to the temperature increase measured by its maximum amplitude $\Delta T_{m}$; and the electrostatic softening linked to the parallel-plate capacitors for sensing. The two contributions to the nonlinear term $K_{3}$ are instead respectively given by: the relaxation of the thermal-induced axial load at second-order, due to the longitudinal stretch of the beam; and once again the softening provided by the parallel-plate capacitor effects. Finally, the external load accounts for the Lorentz effect, as it results proportional to the density $i$ of the current flowing in the beam and to the external magnetic field $B$. 
Relation (3), known as the Duffing equation, provides a maximum amplitude at varying circular frequency $\Omega$ of the forcing term $F=F_{0} \cos \Omega t$ as a solution to [2]:

$$
\left(\frac{F_{0}}{K_{1}}\right)^{2}=\left(2\left(1-\frac{\Omega}{\omega_{0}}\right) \mathcal{V}_{\max }+\frac{3}{4} \frac{K_{3}}{K_{1}} \mathcal{V}_{\max }^{3}\right)^{2}+\left(\frac{d}{m \omega_{0}} \mathcal{V}_{\max }\right)^{2}
$$

where $\omega_{0}=\sqrt{K_{1} / m}$. At resonance, the magnitude of $\mathcal{V}_{\max }$ is:

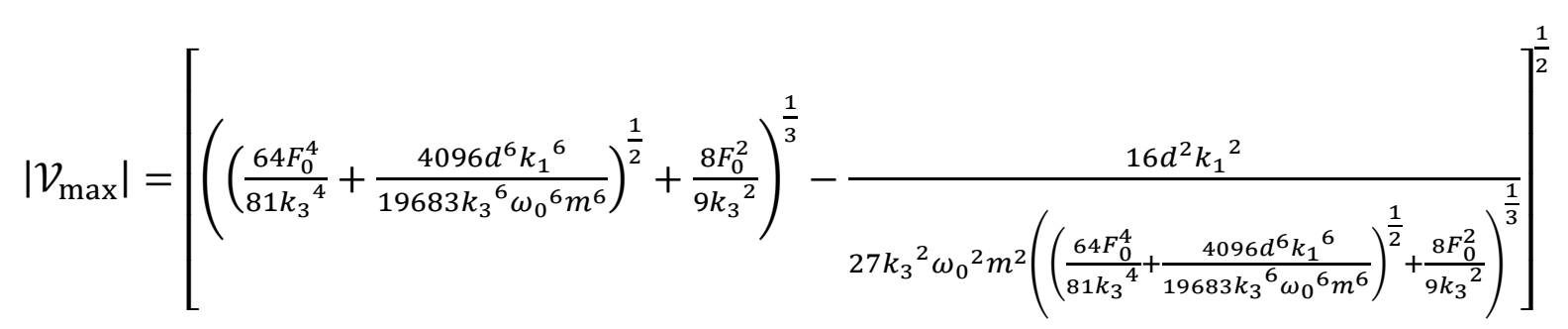

In [2], we proposed a procedure to optimally set the length $L$ and the width $h$ of the beam ( $b$ being assigned due to technological reasons) in order to maximize $\left|\mathcal{V}_{\max }\right|$ and also minimize the relevant power consumption, simultaneously. Starting from the obtained solution of this multi-physics optimization, we assess next the sensitivity of such configuration to imperfections.

\section{Stochastic analysis: sensitivity to imperfections}

Figure 2. Monte Carlo analysis: input statistical distributions (given in terms of occurrences in the analysis) of (a) width $h$ and (b) polysilicon Young's modulus $E$.

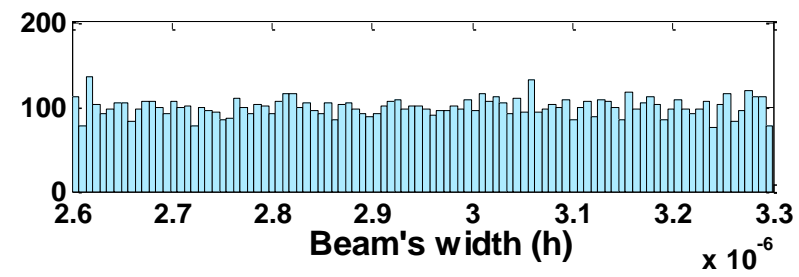

200

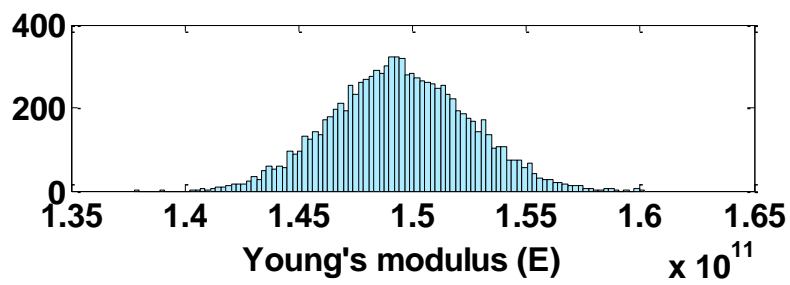

500

$0_{2}$

400

400

200

70
3

al sensiti

In this Section, we focus on the assessment of micro-scale stochastic effects on the device sensitivity, as measured by the amplitude $\left|\mathcal{V}_{\text {max }}\right|$ of the nonlinear oscillations of the resonant structure. Additional effects obviously play a role in defining not only the aforementioned sensitivity of the device, but also all the other performance indexes like, e.g. power consumption, bandwidth, and resolution, see [3]. Such effects are here disregarded to get specific insights into the effects on the sensitivity of so-called micro-scale imperfections, as measured by the scattering of the beam width $h$ and of the Young's modulus $E$ of the polysilicon film constituting the movable structure.

The beam width cannot be known deterministically, as overetch might induce scattering around the target or design value $\bar{h}$. Since information is rather poor concerning the possible pdf of the overetch 
thickness to be adopted in the analysis, we have opted for a uniform distribution in a pre-defined interval $\left[\bar{h}-h_{o}^{-}, \bar{h}+h_{o}^{+}\right]$, where $h_{o}^{-}$and $h_{o}^{+}$were given in the order of $0.15 \mu \mathrm{m}$ on each side of the beam, see Figure 2(a).

Recent analyses [4] showed that, in the case of slender vibrating beams, the polycrystalline morphology may affect much the resulting structural response through its overall Young's modulus. In fact, if the beam width $h$ in on the same length-scale of the characteristic size or diameter $s$ of a single $\mathrm{Si}$ grain, the local arrangement (hence, the film morphology) gives rise to a bending stiffness which cannot be appropriately described through an average, characteristic value only, to be obtained e.g. via asymptotic homogenization. Accordingly, to catch high order statistics of the pdf of $E$, a Monte Carlo analysis has been run to obtain the data reported in Figure 2(b). Due to the target beam width $h=3$ $\mu \mathrm{m}$ and to the standard size $s \cong 0.75 \mu \mathrm{m}$ of the $\mathrm{Si}$ grains, in order to attain objective statistical properties 1,000 numerical homogenization analyses [4] have been run, each one featuring its own film morphology.

Figure 3. Monte Carlo analysis: output statistical distribution of the amplitude $\left|\mathcal{V}_{\max }\right|$ of the oscillations, as a function of (a) width $h$ and (b) Young's modulus $E$. The red lines represent the results provided by the analytical model of Section 2. $\times 10^{-6}$
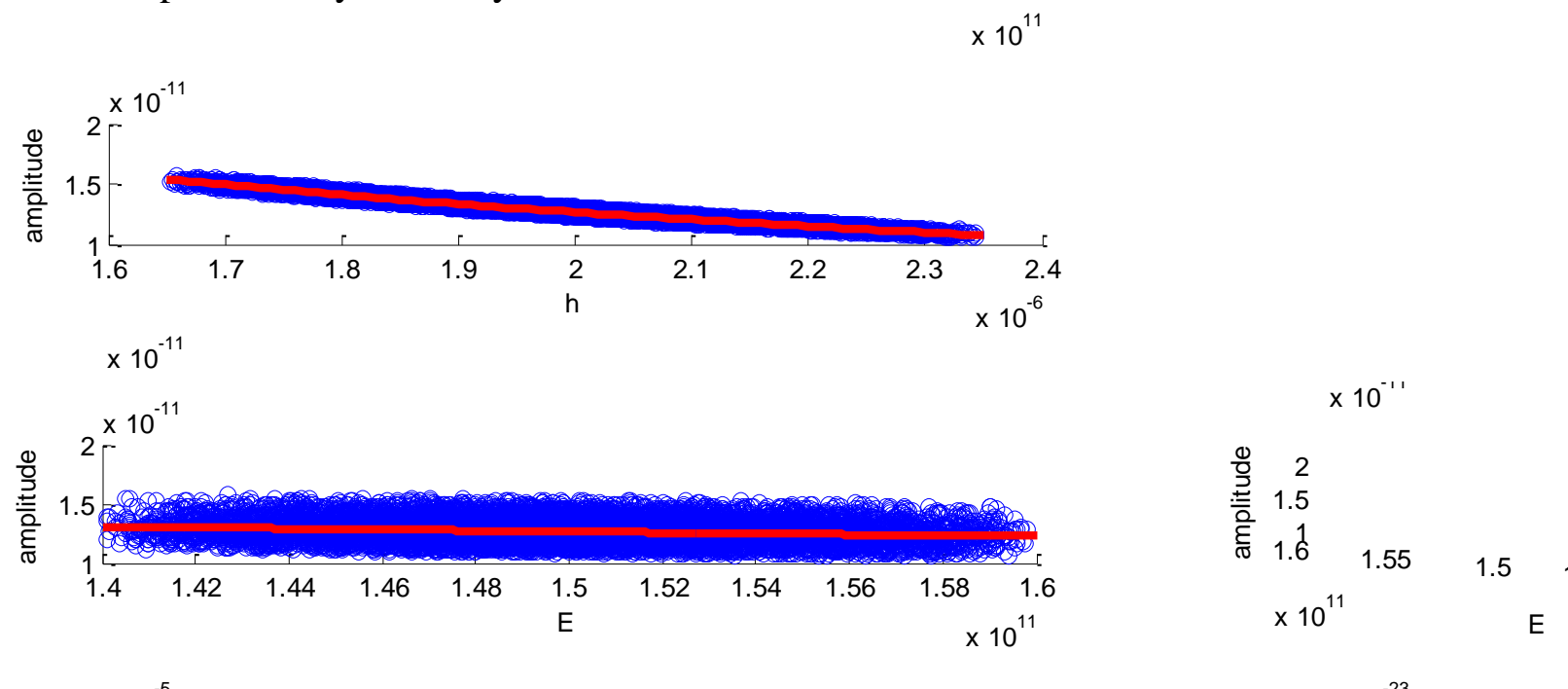

Accounting for the number of realizations adopted in the former homogenization step to build the $E$ pdf, 1,000 analyses have been run here as well. The only approximation adopted in this Monte Carlo analysis was to consider the values of $h$ and $E$, even if scattered, independent of the longitudinal beam coordinate $x$. Considering a variable distribution also function of $x$, would lead to local bending effects not compliant with the assumption, in the analytical model of Section 2, that beam deforms according to its first flexural mode. This topic will be further addressed in future investigations.

Figure 3 gathers the results of the Monte Carlo investigation, in terms of scattering of the amplitude $\left|\mathcal{V}_{\text {max }}\right|$ of the oscillations at varying $h$ and $E$; here each structure realization leads to a single blue circle. Further to the output of the Monte Carlo analysis, the results of the analytical and deterministic model of Section 2 are also reported with the red lines. Besides the matching between the two series of results, it looks worth discussing two details arising from the graphs: the spreading around the average effect of $h$ is smaller than that around $E$; the sensitivity (in these graphs measured by the slope of the 
red lines) to $h$ is higher than the sensitivity to $E$. The former result is induced by the adopted values $h_{o}^{-}$ and $h_{o}^{+}$, compared to the queues of the statistics of $E$ obtained through the Monte Carlo homogenization on a film representative volume. The latter result is instead linked to the physics of the problem at hand, as $E$ affects linearly the stiffness $K_{1}$ in Eq. (4) 3 whereas $h$ affects cubically (through I) the same term.

\section{Conclusions}

In this work, we have discussed a reduced-order model of the resonance dynamics of the movable structure of a Lorentz force MEMS magnetometer. Such model fully accounts for the (weakly) coupled thermo-electro-magneto-mechanics governing the magnetic field-induced vibrations of the structure.

A statistical analysis at the micro-scale has been then developed, in order to assess the sensitivity of the sensor performance to micrometric imperfections, potentially linked to the overetch and to the morphology of the polysilicon film constituting the vibrating structure. A Monte Carlo investigation has been run, using as input a constrained uniform pdf for the beam width and a pdf for the beam Young's modulus as provided by a statistical homogenization over a sample of the polycrystalline film. Results of the analysis have been shown to well agree with the outcomes of the aforementioned deterministic reduced-order model, which indeed provides only average information on the device performance.

\section{Acknowledgments}

The financial support by STMicroelectronics through the Material Reliability project is gratefully acknowledged.

\section{References and Notes}

1. Li, M.; Rouf, V.T.; Thompson, M.J.; Horsley, D.A. Three-axis Lorentz-force magnetic sensor for electronic compass applications. Journal of Microelectromechanical Systems 2012, 21, 10021010.

2. Bagherinia, M.; Bruggi, M.; Corigliano, A.; Mariani, S.; Lasalandra, E. Geometry optimization of a Lorentz force, resonating MEMS magnetometer. Microelectronics Reliability 2014, to appear.

3. Bagherinia, M.; Corigliano, A.; Mariani, S.; Horsley, D.A.; Li, M.; Lasalandra, E. An efficient earth magnetic field MEMS sensor: modelling and experimental results. Proceedings MEMS IEEE 2014, 26-30 January 2014, San Francisco, CA, pp. 700-703.

4. Mariani, S.; Martini, R.; Ghisi, A.; Corigliano, A.; Beghi, M. Overall elastic properties of polysilicon films: a statistical investigation of the effects of polycrystal morphology. International Journal for Multiscale Computational Engineering 2011, 9, 327-346.

(C) 2014 by the authors; licensee MDPI, Basel, Switzerland. This article is an open access article distributed under the terms and conditions of the Creative Commons Attribution license (http://creativecommons.org/licenses/by/3.0/). 